\title{
Diagnostic contribution of focal cortical dysplasia MRI imaging findings and ADC values
}

\author{
Aydın Aslan", Muhammed Akif Deniz ${ }^{*}$, Zelal Taş Deniz ${ }^{1}$, Mehmet Turmak² and Faysal Ekici ${ }^{3}$
}

\begin{abstract}
Background: Focal cortical dysplasia (FCD) is a condition that often interferes with the cranial mass. Knowledge of focal cortical dysplasia magnetic resonance imaging (MRI) characteristics is of utmost importance for diagnosis. This study aimed to determine the diagnostic contribution of cranial MRI and the apparent diffusion coefficient (ADC) in FCD.

Results: Cranial MRI revealed subcortical hyperintensity $(\mathrm{SCH})$ in 52 patients (82.5\%), blurring of the gray-white matter (GWM) interface (blurring) in 52 patients (82.5\%), cortical thickening (CT) in 48 patients (76\%), cortical signal increase (CSI) in 41 patients (65\%), and transmantle sign (TMS) in 29 patients (46\%). All of the FCDs had a diffusion increase in diffusion-weighted images (DWIs). The mean ADC value at the lesion level was $1.087 \times 10^{-3} \mathrm{~mm}^{2} / \mathrm{s}$ $\left(0.82 / 1.316 \times 10^{-3} \mathrm{~mm}^{2} / \mathrm{s}\right)$, which was significantly higher than the mean ADC value measured from the contralateral symmetric region $\left(0.758 \times 10^{-3} \mathrm{~mm}^{2} / \mathrm{s}, 0.678 / 0.872 \times 10^{-3} \mathrm{~mm}^{2} / \mathrm{s}, p=0.001\right)$.

Conclusion: Results of this study revealed that the most common MRI findings in FCD patients are $\mathrm{SCH}$, blurring, and $C T$. These findings aid in an easier diagnosis in patients with suspected FCD. All of the lesions studied here in had a diffusion increase in DWI. The quantitative mean ADC values detected in the differential diagnosis of other lesions with a diffusion increase can be used as a reference. Therefore, in addition to the ADC values and electroencephalography (EEG) findings, the conventional MRI findings of FCD, which is resistant to medical treatment, can help to facilitate the diagnosis of FCD, which can be treated with surgery.
\end{abstract}

Keywords: Focal cortical dysplasia, Magnetic resonance imaging, Apparent diffusion coefficient

\section{Background}

Focal cortical dysplasia (FCD) is a heterogeneous form of cortical lesions. Literature defines FCD as a malformation of cortical development, cortical dysplasia, cortical dysgenesis, or neuronal migration disorder. Although the term "cortical dysplasia" accounts for many different focal malformations of cortical development, including heterotrophy and polymicrogyria, FCD is commonly used to describe malformations as described by Taylor [1].

FCD consists of the following various types of changes:

- Cortical architectural anomalies:

\footnotetext{
* Correspondence: makifdeniz@yahoo.com

'Department of Radiology, Health Science University Gazi Yaşargil Education Research Hospital, 21090 Diyarbakır, Turkey

Full list of author information is available at the end of the article
}

Columnar disorganization, laminar disruption (changes in the six-layer tangential structure of the cortex)

- Cytological abnormalities:

Hypertrophic neuronal cells: other than those at the typical position at the fifth layer.

Immature neurons: round or oval cells with large nuclei that are not present in the mature cortex.

Dysmorphic neuron: abnormal size and morphology of axons and dendrites, in addition to an increased accumulation of neurofilament proteins.

Balloon cells: these cells are pathognomonic for Taylor type dysplasia [1]. They generally have the immunohistochemical properties of neuronal and glial cells, including a poorly defined membrane, single or 
Table 1 New classification system of focal cortical dysplasia by Blumcke et al. 2011

\begin{tabular}{|c|c|}
\hline Type & Characteristic features \\
\hline । & $\begin{array}{l}\text { - a-Focal cortical dysplasia with abnormal radial cortical lamination } \\
\text { - b-Focal cortical dysplasia with abnormal tangential 6-layer cortical lamination } \\
\text { - c-Focal cortical dysplasia with abnormal radial and tangential cortical lamination }\end{array}$ \\
\hline$\|$ & $\begin{array}{l}\cdot \text { a_Focal cortical dysplasia with dysmorphic neurons } \\
\cdot \text { b-Focal cortical dysplasia with dysmorphic neurons and balloon cells }\end{array}$ \\
\hline III & $\begin{array}{l}\text { - a-Architectural distortion of cortical layer in temporal lobe with hippocampal atrophy } \\
\text { - b-Architectural distortion of cortical layer adjacent to glial or glioneuronal tumor } \\
\text { - c-Architectural distortion of cortical layer adjacent to vascular malformation } \\
\text { - d-Architectural distortion of cortical layer adjacent to other lesions acquired in } \\
\text { early childhood such as trauma, ischemic event, encephalitis }\end{array}$ \\
\hline
\end{tabular}

multiple eccentric nuclei, and are large cells with an eosinophilic cytoplasm [2].

FCD was first differentiated from developmental malformations in ten patients by Taylor et al. in 1971, and since then, its classification has undergone various modifications. The palmini classification, which was modified by Blumcke in 2011, has been used most recently (Table 1 ).

FCD is frequently discovered during neuropathological examinations of pediatric patients undergoing surgery (especially those surgeries due to drug-resistant epilepsy), and it is one of the most common causes of partial epilepsy that can be treated with surgery $[3,4]$. Gender does not play a role in the development of FCD. The absence of a known genetic cause for mild cortical malformations suggests that their etiology may be due to an external factor that influences the migration and differentiation stages of neurons during cortical development.

The etiology of FCD is not well understood. While it is responsible for approximately half of the drug-resistant epilepsy cases in children and adults, patients with FCD often respond well to treatment.

The continuous improvement of neuroimaging techniques has led to more accurate evaluations of pathological lesions, which creates a better chance that appropriate patients will be recommended for surgical treatment.

Because there has been a rapid development in the fields of genetics and diagnostics, FCD should be reviewed by these disciplines, which may lead to an increased number of treatment methods for this disease.

\section{MRI findings}

Magnetic resonance imaging (MRI) is the most common imaging method used to assess brain pathology in FCD.

MRI findings in FCD depend on both the size and the characteristics of the lesions. For example, FCD type Ia causes mild hemispheric hypoplasia without other visible lesions $[5,6]$. In FCD type IIb, there are localized areas with increased cortical thicknesses and signs of blurred funnel-shaped regions, which indicates gray-white matter separation at the bottom of the sulcus (transmantle MR sign). Lesion signal intensity in FCD is agedependent. In neonates and infants, lesion signal intensity in FCD type IIb is hyperintense in T1A images and mildly hypointense in T2A images. In older patients, lesion signal intensity has a hyperintense wedge-shape extending to the subcortical and deep white matter in FCD $\mathrm{T} 2$ and FLAIR images. In some cases, the subcortical

Table 2 Parameters used in 3-Tesla MRI

\begin{tabular}{|c|c|c|c|c|c|}
\hline & Axial T2 sequence & Coronal T2 sequence & Axial FLAIR sequence & Coronal FLAIR sequence & Sagittal T1A 3D FFE sequence \\
\hline TR/TE & $2500 / 80$ & $3000 / 80$ & $10000 / 125$ & $11000 / 120$ & $8.1 / 3.7$ \\
\hline IR (msn) & - & - & 2800 & 2800 & 1006 \\
\hline FOV (mm) & 230 & 220 & 230 & 220 & 240 \\
\hline Matrix & $400 / 255$ & $336 / 210$ & $352 / 198$ & $336 / 210$ & $240 / 240$ \\
\hline Section thickness & 5 & 3 & 5 & 3 & 1 \\
\hline FA (degree) & - & - & - & - & 8 \\
\hline Section space & 1 & 1 & 1 & 2 & 1 \\
\hline Viewing time & $2 \min 15 \mathrm{~s}$ & $4 \min 21 \mathrm{~s}$ & $4 \min 0 \mathrm{~s}$ & $4 \min 21 \mathrm{~s}$ & $7 \mathrm{~min} 29 \mathrm{~s}$ \\
\hline
\end{tabular}


Table 3 Parameters used in 1.5-Tesla MRI

\begin{tabular}{|c|c|c|c|c|c|}
\hline & Axial T2 sequence & Coronal T2 sequence & Axial FLAIR sequence & Coronal FLAIR sequence & Sagittal T1 3D FFE sequence \\
\hline TR/TE & $4400 / 100$ & $2200 / 100$ & $6000 / 120$ & $8000 / 125$ & $7 / 3$ \\
\hline IR (msn) & - & - & 2000 & 2450 & 1006 \\
\hline $\mathrm{FOV}(\mathrm{mm})$ & 230 & 210 & 230 & 220 & 240 \\
\hline Matrix & $300 / 220$ & 204/159 & $256 / 168$ & 193/156 & $256 / 256$ \\
\hline Section thickness & 5 & 3 & 4 & 3 & 1 \\
\hline FA (degree) & - & - & - & - & 8 \\
\hline Section space & 1 & 0.3 & 1 & 0.3 & 1 \\
\hline Viewing time & $1 \mathrm{~min} 40 \mathrm{~s}$ & $1 \mathrm{~min} 55 \mathrm{~s}$ & $3 \min 0 \mathrm{~s}$ & $4 \mathrm{~min} 0 \mathrm{~s}$ & $6 \min 0 \mathrm{~s}$ \\
\hline
\end{tabular}

linear or curvilinear T2/FLAIR hyperintensity focus extends to the superolateral margin of the lateral ventricle $[5,6]$.

In FCD type IIb, contrast does not hold in T1contrasted series. Increased diffusion and reduced fraction anisotropy (FA) are seen in FCD type IIb diffusion-weighted images (DWI). Reduced $\mathrm{N}$-acetylaspartate:creatinine ratio (NAA:Cr) and increased Myoinositol $(\mathrm{ml})$ rates can be monitored via magnetic resonance spectroscopy (MRS). Normal or decreased cerebral blood flow (rCBV) is seen in perfusion MR [7].

FCD type III has been recently identified and is primarily encountered in patients with hippocampal sclerosis. FCD type IIIa is characterized by volume loss in the anterior temporal lobe with abnormal white matter hyperintensity on $\mathrm{T} 2$ and FLAIR images, while the cortex appears normal from other views [8].

Voxel-based morphometry and statistical parametric mapping are advanced techniques that can enhance the detection of epileptogenic lesions in patients with negative tissue analysis as determined by standard MR images [9].

The current study aimed to determine the prevalence of previously described MR imaging criteria for FCD, to identify MR imaging findings (with the goal of optimizing the detection of FCD in clinical practice), to measure the mean $\mathrm{ADC}$ values, and to compare these values with those of cortical-subcortical lesions to determine whether this parameter will be useful in the differential diagnosis of FCD.

\section{Materials and methods \\ Patients}

In this study, we retrospectively reviewed the cranial MRI images of 63 patients (33 males, 30 females) who were suspected of having FCD based on clinical findings (ictal-video electroencephalography (EEG)) and MRI findings, who were resistant to medical treatment, and who were seen in our clinic between February 2011 and July 2016 . The age range of the patients was $1-60$ years (the mean age was $18.92 \pm 16.13$ years in males and $25.41 \pm 11.79$ years in females).

Patients with cerebellar cortical dysplasia, artifacts, and lesions detected in the vicinity of FCD, or patients with suspected FCD in MRI but who were not clinically diagnosed with FCD were excluded from this study.

This study was approved by the ethics committee of our university.

\section{MRG protocol}

Brain MR scans were performed with 1.5-T and 3.0-T MRI devices (Achieva; Philips Medical Systems, Best, the Netherlands) using an eight-channel cranial coil. All examinations were performed according to the epilepsy protocol. The MRI protocol routinely used for epilepsy

Table 4 FCD anatomic distribution

\begin{tabular}{llllllll}
\hline & Frontal & Parietal & Temporal & Parahippocampal & Insular & Hemisphere & Total \\
\hline Right & 17 & 2 & 3 & 0 & 1 & 2 & 25 \\
Left & 16 & 7 & 4 & 2 & 0 & 1 & 32 \\
Bilaterally & 5 & 0 & 0 & 2 & 4 & 3 & 6 \\
Total & 38 & 9 & 7 & 0 & 3 \\
\hline
\end{tabular}


Table 5 Distribution of FCD cranial MRI findings

\begin{tabular}{lll}
\hline & Frequency $(n)$ & $\%$ \\
\hline Cortical thickening (CT) & 48 & 76.2 \\
Cortical signal intensity (CSI) & 41 & 65.1 \\
Blurring in the gray-white matter (GWM) interface (blurring) & 52 & 82.5 \\
Subcortical white matter hyperintensity (SCH) & 52 & 82.5 \\
Transmantle sign (TMS) & 29 & 46 \\
\hline
\end{tabular}

in our hospital includes T2-weighted FSE (fast spin echo) on the axial and coronal planes, fluid-attenuated inversion recovery (FLAIR), and non-contrast T1-weighted 3D turbo field echo (TFE) sequences (Tables 2 and 3). During the examination, T1weighted axial, coronal, and sagittal plane images were obtained in 3D TFE sequence using intravenous paramagnetic contrast material (gadodiamide [Omniscan; Amersham Health, Cork, Ireland], gadopentetate dimeglumine [Magnevist; Schering AG, Berlin, Germany], and gadobutrol [Gadovist; Schering AG, Berlin, Germany]) at a dose of $0.1 \mathrm{mmol} / \mathrm{kg}$ through the antecubital vein at a rate of $2 \mathrm{ml} / \mathrm{s}$ based on determined pathology.

The cranial MRI images of all patients who were accepted as having FCD via cranial MRI and clinical data (ictal-video EEG) were examined retrospectively by two neuroradiologists (with 5 and 8 years' experience).

The following five semiologic findings were analyzed:

1. Cortical thickening (CT): thickening of the normal cortex by $50 \%$ in at least two sequences (T1WI and T2WI)

2. Cortical signal intensity (CSI): signal change involving the entire thickness of T1WI, T2WI, and/ or FLAIR cortex

3. Blurring in the gray-white matter (GWM) interface (blurring): signal change in at least one sequence and two planes

4. Subcortical white matter hyperintensity $(\mathrm{SCH})$ : abnormal signal change in the subcortical white matter according to the normal cortex

5. Transmantle sign (TMS): signal change extending from the subcortical white matter to the ventricle in T1WI and T2WI sequences
The DWI images of the lesions detected in routine MRI were analyzed using the Philips Extended MR Workspace. Since the DWI has poor resolution, there may be some difficulties in lesion imaging. In order to prevent this, the lesion should be identified with both conventional and dynamic MRI and evaluated accordingly. A round or oval ROI was placed manually over the lesion to cover at least $50 \%$ of the lesion size using b:0 images, which have better anatomical detail compared to other maps. The mean ADC values in these areas were calculated directly on the ADC maps.

The mean ADC measurements were calculated for each patient using the same ROI areas as follows:

1. On the cortical, subcortical visible hyperintense lesion area

2. From the contralateral symmetric healthy parenchyma

Cerebrospinal fluid (CSF) was not included in the measurements in order to prevent false measurements.

\section{Statistical analysis}

All data were analyzed using the SPSS 18.0 package program. The Shapiro-Wilk test was used to determine the normality of lesions as well as the mean $\mathrm{ADC}$ and contralateral symmetric healthy ADC values. For paired comparisons, we used the parametric "Student's $t$ test" for groups with a normal distribution and the "Mann-Whitney $U$ test" for groups with a non-normal distribution. The ADC values of the groups with and without lesions were compared according to the test results. Values of $p<0.05$ were accepted as significant.

Table 6 ADC values obtained from lesional and symmetric healthy parenchyma

\begin{tabular}{lllll}
\hline & $N$ & Mean ADC & Standard deviation & Min/Max ADC \\
\hline Lesion & 63 & $1.087 \times 10^{-3} \mathrm{~mm}^{2} / \mathrm{s}$ & $0.15 \times 10^{-3} \mathrm{~mm}^{2} / \mathrm{s}$ & $0.82 / 1.316 \times 10^{-3} \mathrm{~mm}^{2} / \mathrm{s}$ \\
Normal parenchyma & 63 & $0.758 \times 10^{-3} \mathrm{~mm}^{2} / \mathrm{s}$ & $0.052 \times 10^{-3} \mathrm{~mm}^{2} / \mathrm{s}$ & $0.678 / 0.872 \times 10^{-3} \mathrm{~mm}^{2} / \mathrm{s}$ \\
\hline
\end{tabular}



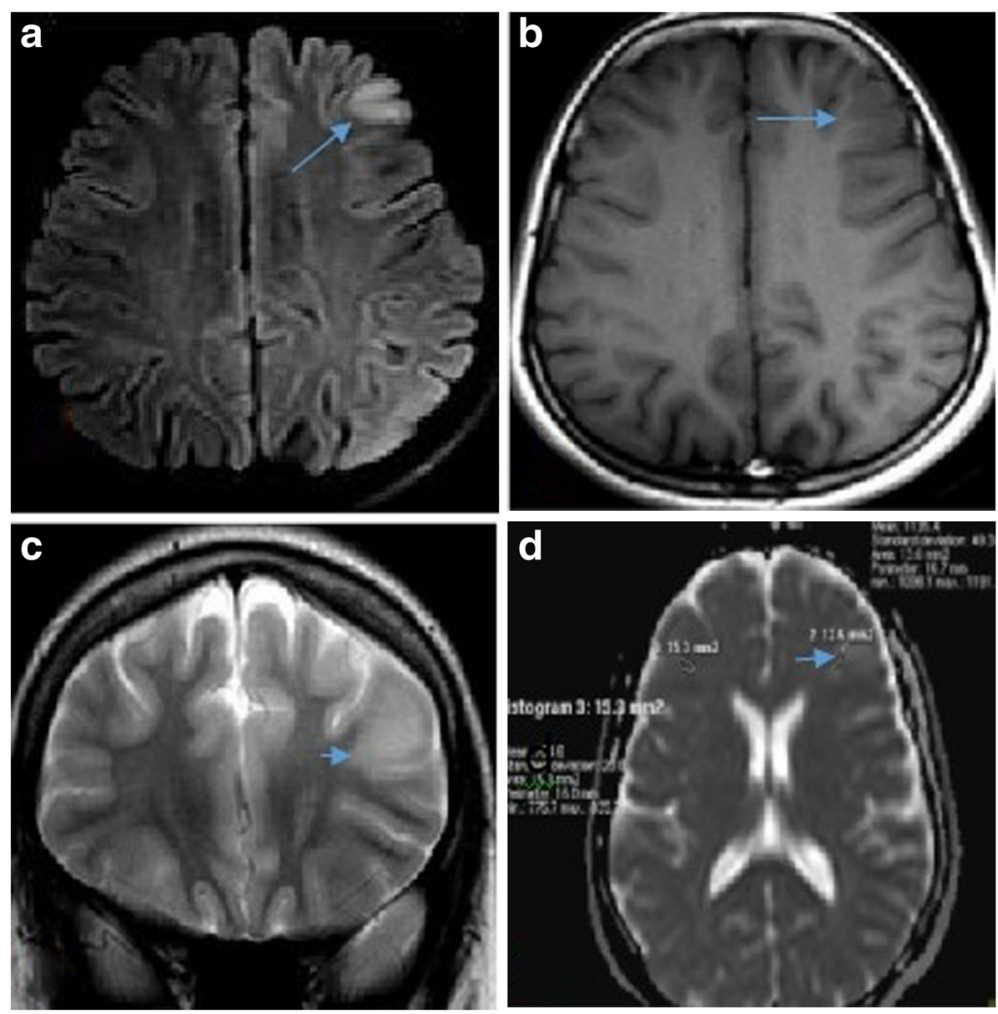

Fig. 1 A 48-year-old male patient with epilepsy. a Increase in cortical signal intensity in axial FLAIR sequence-subcortical signal intensity. b Cortical thickening in axial T1A sequence. $\mathbf{c}$ Cortical signal intensity in coronal T2A sequence, blurring in white-gray matter interface. $\mathbf{d}$ Hyperintense area in axial $\mathrm{dADC}$ sequence, $\mathrm{ADC}$ measurement from this area and from a symmetrical area

\section{Findings}

The male/female ratio of the 63 patients was 1.1 (male = 33; female $=30$ ). The localization of FCD was frontal lobe in $38(60 \%)$ patients, parietal lobe in nine (14\%) patients, temporal lobe in seven (11\%) patients, insular cortex in four (6\%) patients, parahippocampal gyrus in two (3\%) patients, and hemispheric area in three $(4.7 \%)$ patients.

A total of 25 lesions (39.5\%) were located in the right hemisphere (frontal lobe in 17 patients, parietal lobe in two patients, temporal lobe in three patients, insular cortex in one patient, hemisphere in two patients), 32 lesions (50.7\%) were located in the left hemisphere (frontal lobe in 16 patients, parietal lobe in seven patients, temporal lobe in four patients, insular cortex in two patients, parahippocampal in two patients, and hemisphere in one patient), and six lesions $(9.5 \%)$ were located bilaterally (frontal lobe in five patients and insular cortex in one patient) (Table 4).

In the current study, 20 patients (32\%) had all five of the cranial MRI findings. We found CT-CSI in 40 patients (63\%), blurring-SCH in 50 patients (79\%), and blurring SCH-TMS in 29 patients (46\%).
CT was limited to the small cortical area in 48 patients (76\%), and it was not more than twice the normal cortex thickness.

CSI was present in 41 patients (65\%). Forty of these patients (97.5\%) also had CT.

Blurring was present in 52 patients $(82.5 \%)$. Of these patients, 50 (96\%) had $\mathrm{SCH}$.

$\mathrm{SCH}$ was detected in 52 patients $(82.5 \%)$. Of these patients, 50 (96\%) had blurring.

TMS was present in 29 patients (46\%). All of these patients had SCH (100\%). All of the FCD cranial MRI findings are summarized in Table 5.

All of the lesions presented with a diffusion increase in DWI. The FCD mean ADC values were $1.087 \times 10^{-3}$ $\mathrm{mm}^{2} / \mathrm{s}\left(0.82-1.316 \times 10^{-3} \mathrm{~mm}^{2} / \mathrm{s}\right)$, and the symmetrical normal parenchyma mean ADC was $0.758 \times 10^{-3}$ $\mathrm{mm}^{2} / \mathrm{s}\left(0.678-0.872 \times 10^{-3} \mathrm{~mm}^{2} / \mathrm{s}\right)$. The normalities of the mean ADC values of the lesions and of the contralateral healthy parenchyma were investigated using the Shapiro-Wilk test, which indicated that both groups were normally distributed $(p<0.05)$. The Student's $t$ test revealed a significant difference between the mean ADC values of the groups with and without lesions $(p=0.001)$. 

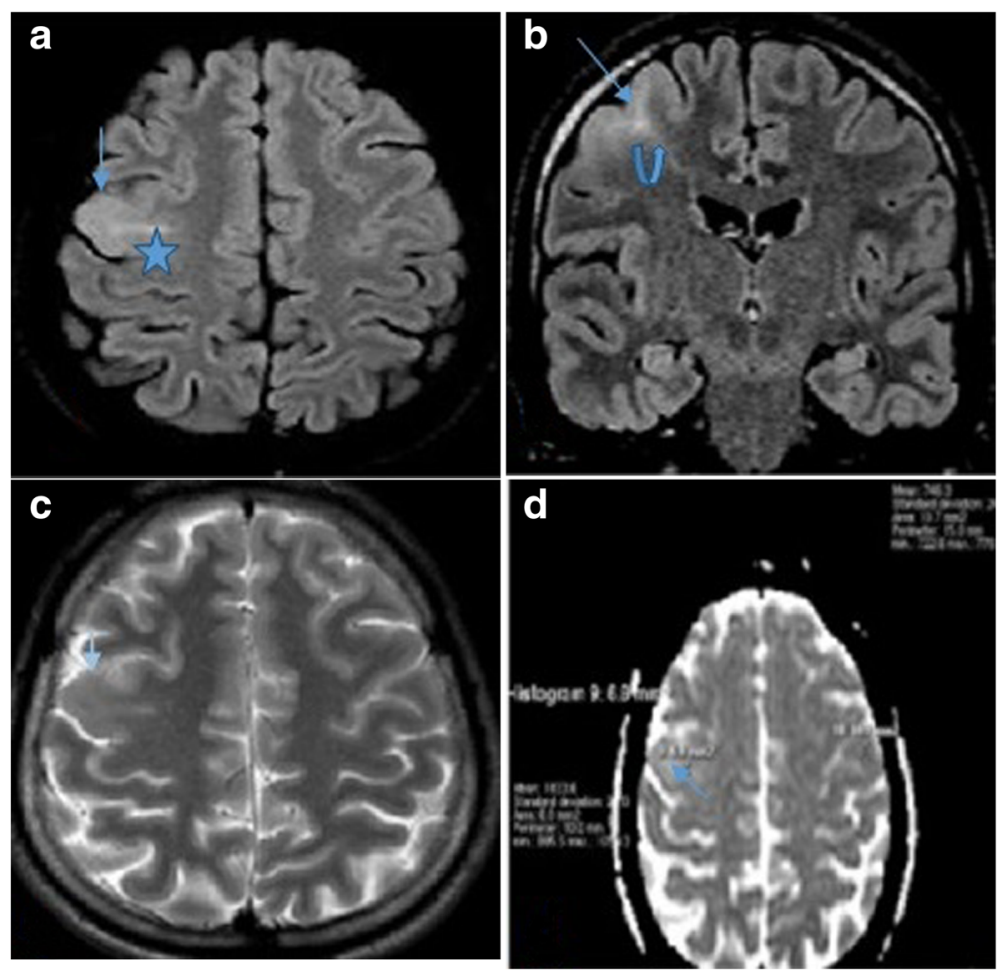

Fig. 2 A 35-year-old male patient with epilepsy. a Cortical signal change in axial FLAIR sequence-blurring in GW matter interface (arrow) and transmantle sign (star). b Cortical signal change in coronal FLAIR sequence-blurring in GW matter interface (arrow) and transmantle sign (thick arrow). c Increase in cortical signal intensity-blurring in GW matter interface. $\mathbf{d}$ Hyperintense area in axial dADC sequence, ADC measurement from this area and from a symmetrical area

The average ADC (c-ADC) values, which were measured from the cortical and subcortical hyperintense areas and contralateral symmetric healthy areas in 63 patients with FCD, are summarized in Table 6 (Figs. 1, 2, 3, 4 and 5).

\section{Discussion}

MRI is the most commonly used imaging method for evaluating brain pathology in FCD. Cranial MR imaging usually shows abnormalities in FCD type 2, but only shows abnormalities in some FCD type 1 cases [1]. Despite the use of appropriate protocols in specialist centers, MRI cannot be used to diagnose type 2b FCD in $50 \%$ of patients, which can lead to delays in treatment [4]. Despite severe and persistent epilepsy, in most series, the average time from the onset of seizures to surgery is about 1 year [5-8]. Previous studies have identified typical MR imaging features such as cortical anomalies (thickening, increase in T2 signal), subcortical white matter (blurring in graywhite matter interface), increase in subcortical signal, and transmantle sign [10-21]. Earlier reports of MR imaging findings were based on a limited number of patients [12, 15]. However, the newer and more extensive series have not provided a comprehensive analysis of all reported MR imaging findings, and the prevalence of each finding has varied considerably between studies (Table 7). In addition, the ratio of FCD-negative MR imaging findings has varied significantly due to differences in imaging protocols and selection bias.

In the current study, which investigated the cranial MRI findings of FCD, the systematic qualitative basic MRI findings are as follows: 20 patients (32\%) had all five of the cranial MRI findings. There was cortical thickening and an increase in cortical signal intensity in 40 patients $(63 \%)$, subcortical hyperintensity and blurring in the gray-white matter interface in 50 patients (79\%), and blurring in the gray-white matter interface, subcortical hyperintensity, and transmantle sign in 29 patients (46\%).

Cortical thickening, subcortical hyperintensity, and blurring in the GW matter interface are considered major findings in terms of the MRI diagnosis of FCD.

The increase in cortical signal intensity is a wellknown finding of FCD. In our current study, this was found in $65 \%$ of the patients, while other studies have 

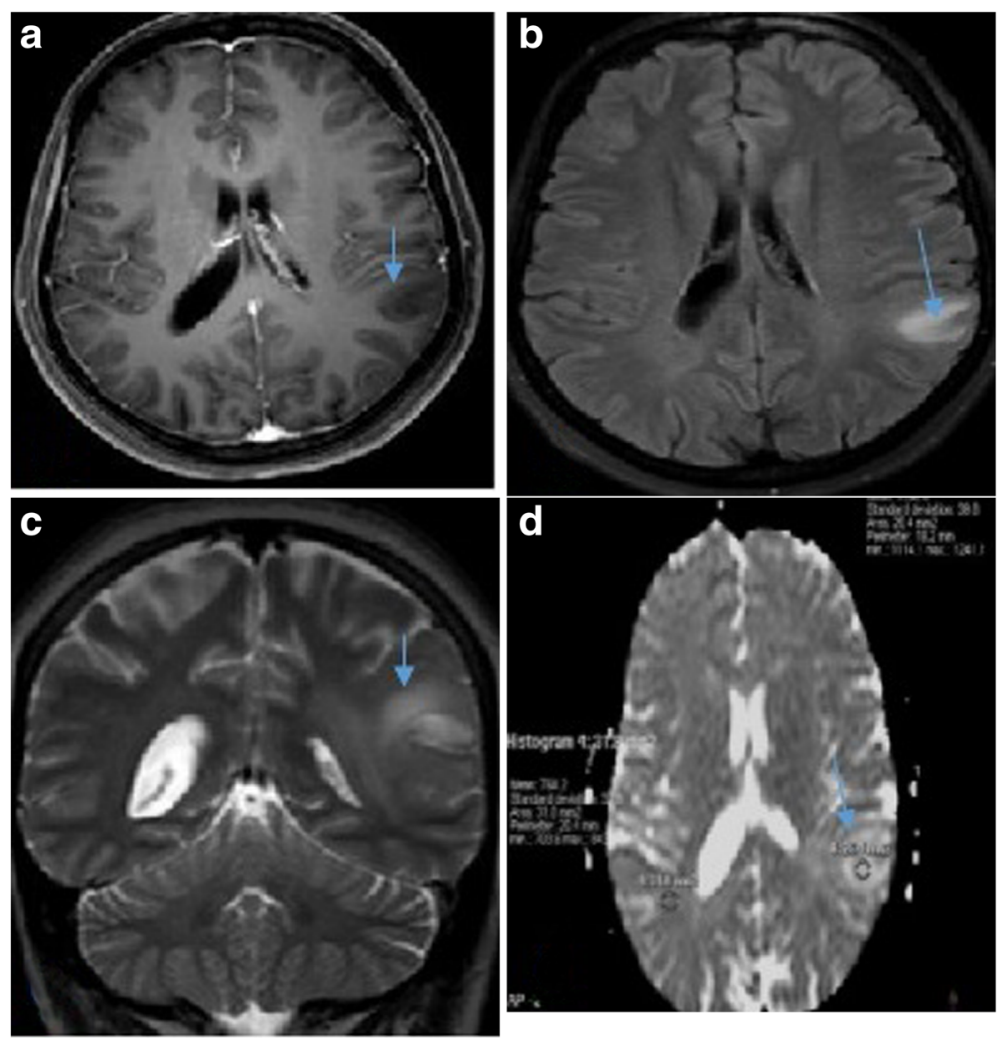

Fig. 3 A 36-year-old female patient with epilepsy. a Cortical thickening in axial T1A sequence. b Subcortical hyperintense in axial FLAIR sequenceblurring in GW matter interface. $\mathbf{c}$ Increase in cortical signal intensity in coronal T2A sequence-blurring in GW matter interface. $\mathbf{d}$ Hyperintense area in axial $\mathrm{AADC}$ sequence, $\mathrm{ADC}$ measurement from this area and from a symmetrical area

reported the increase in cortical signal intensity was found at rates between 15 and $62 \%$ [10, 11, 13, 15, 23]. This finding was more obvious in FLAIR sequences. With the increased use of 3D FLAIR and high-field MR imaging, we believe that the cortical signal intensity will be more easily detected and more reliable in the future [23].

The transmantle sign was positive in $46 \%$ of the patients in our current study, while previous studies report this range between 20 and $83 \%$ [10, 11, 13, 15, 23]. This wide range suggests that the transmantle sign was either ignored or combined with change in the subcortical signal in some studies. It has been reported that the transmantle sign is a specific feature for cortical development malformations [18]. The transmantle sign is also associated with the presence of hypomyelination and balloon cells in white matter underlying the dysplastic lesion [12, 24]. It may also occur in other developmental abnormalities such as venous or arteriovenous malformations, and is not specific in FCD [25]. However, its relationship with cortical thickening and blurring in GW matter is an important sign for the diagnosis of FCD.
In a study conducted with 71 patients (male/female ratio of 1:3) who were diagnosed with FCD type $2 \mathrm{~b}$ using histopathological correlation, there was a left/right hemisphere ratio of $1: 35$, and lobar distribution was as follows: frontal lobe $(n=60)$, parietal lobe $(n=7)$, temporal lobe $(n=2)$, and occipital lobe $(n=2)$. Moreover, subcortical hyperintensity and blurring in GW matter interface was $90 \%$, transmantle sign was $83 \%$, cortical thickening was $71 \%$, and cortical signal increase was $50 \%$. Subcortical signal changes were detected as major findings [23]. In our current study (male/female ratio of $1: 1$ ), the left/right hemisphere ratio was $1: 28$, and lobar distribution was as follows: frontal lobe $(n=$ $38)$, parietal lobe $(n=9)$, and temporal lobe $(n=7)$. Our current findings were similar to those in the literature in terms of gender, hemispheric distribution, and lobar distribution. Although the subcortical signal changes in our current study were detected at lower rates than those found in the literature, the subcortical signal changes with cortical thickening were detected as major findings. The difference 

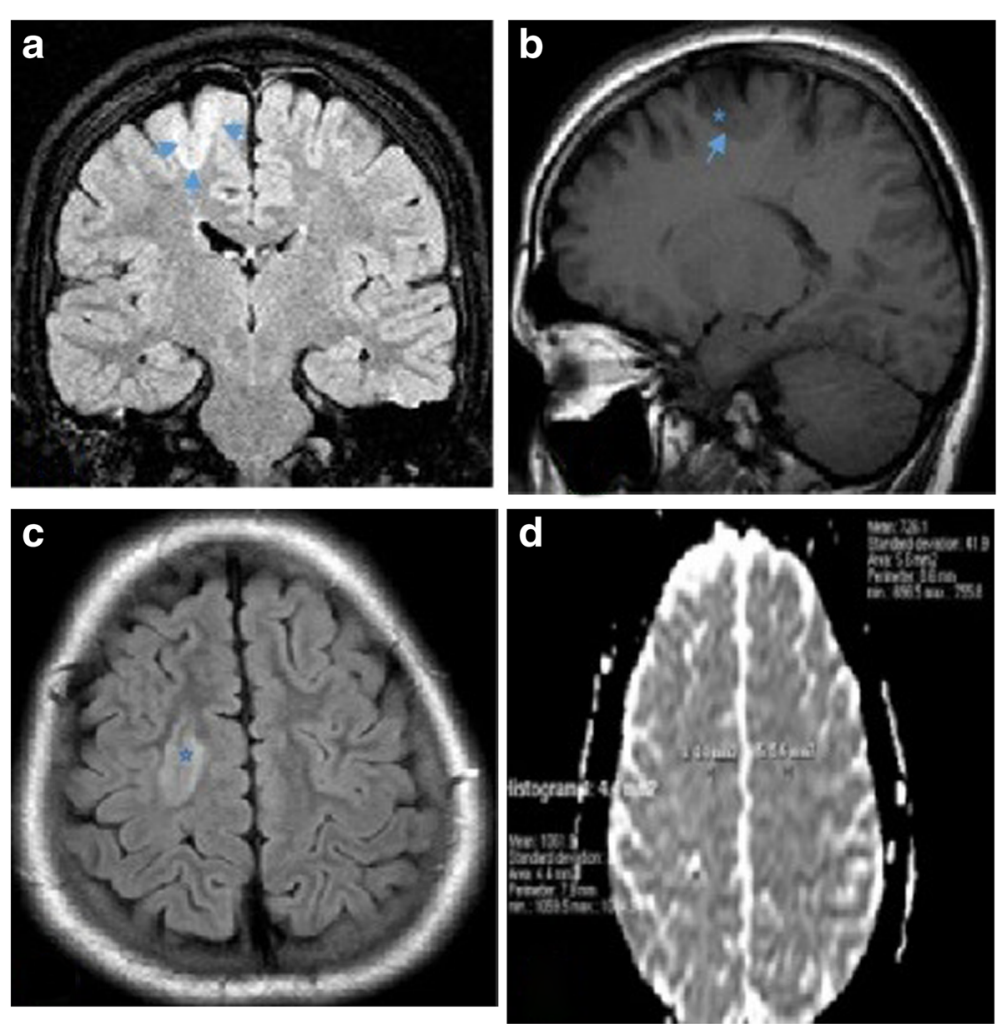

Fig. 4 A 13-year-old male patient with epilepsy. a Subcortical hyperintense in coronal FLAIR sequence. b Cortical thickening in sagittal T1A sequence. $\mathbf{c}$ Increase in cortical signal intensity in axial FLAIR sequence. $\mathbf{d}$ Hyperintense area in axial dADC sequence, ADC measurement from this area and from a symmetrical area

between the rates of subcortical signal changes in our current study and in the literature may be due to the fact that our current study only included patients with positive MRI findings.

The findings of frontal lobe dominance in FCD localization and the rare occurrence of lesions in the temporal lobe in our current study are comparable with those in the literature $[14,15,22]$. Other epileptogenic lesions are located predominantly in the temporal lobe [23]. This increases the suspicion that FCD, one of the most common causes of drug-resistant partial epilepsy, may be located in the frontal lobe. This hypothesis has been supported by cryptogenic partial epilepsy series, in which $40 \%$ of resected cortical lesions (especially in the frontal lobe) were type 2 FCD via histology [26-28].

$\mathrm{ADC}$ values are the quantitative correspondence of diffusion in biological tissues. The movement of water molecules throughout tissue is influenced by the tissue's cellularity and histological structure. These parameters affect diffusion and perfusion, and thus ADC values [29]. Low ADC values are indicative of restricted diffusion due to hypercellularity [30].
In our current study, there was a significant difference between lesional and contralateral symmetric healthy parenchyma ADC values $(p<0.001)$. The mean ADC value at lesion level was $1.087 \times 10^{-3}$ $\mathrm{mm}^{2} / \mathrm{s}\left(0.82 / 1.316 \times 10^{-3} \mathrm{~mm}^{2} / \mathrm{s}\right)$ and the symmetrical normal parenchyma mean ADC value was 0.758 $\times 10^{-3} \mathrm{~mm}^{2} / \mathrm{s}\left(0.678 / 0.872 \times 10^{-3} \mathrm{~mm}^{2} / \mathrm{s}\right)$. FCD may be confused with low-grade astrocytomas with cortical involvement. The mean ADC values reported in the literature for low-grade astrocytomas are quite variable [31-33] (Table 8). Although the mean ADC values obtained in our current study differ from some in the literature, it may not always be reliable to distinguish FCD from other lesions by looking only at mean ADC values. The mean ADC values with conventional MRI findings may contribute to a diagnosis.

In our current study, which evaluated patients diagnosed with FCD both radiologically and clinically, major limitations include a lack of histopathologic correlation, a lack of inclusion of MRI negative patients, and a lack of exclusion of lesions that may be confused with FCD. Furthermore, the specificity of MR imaging findings 

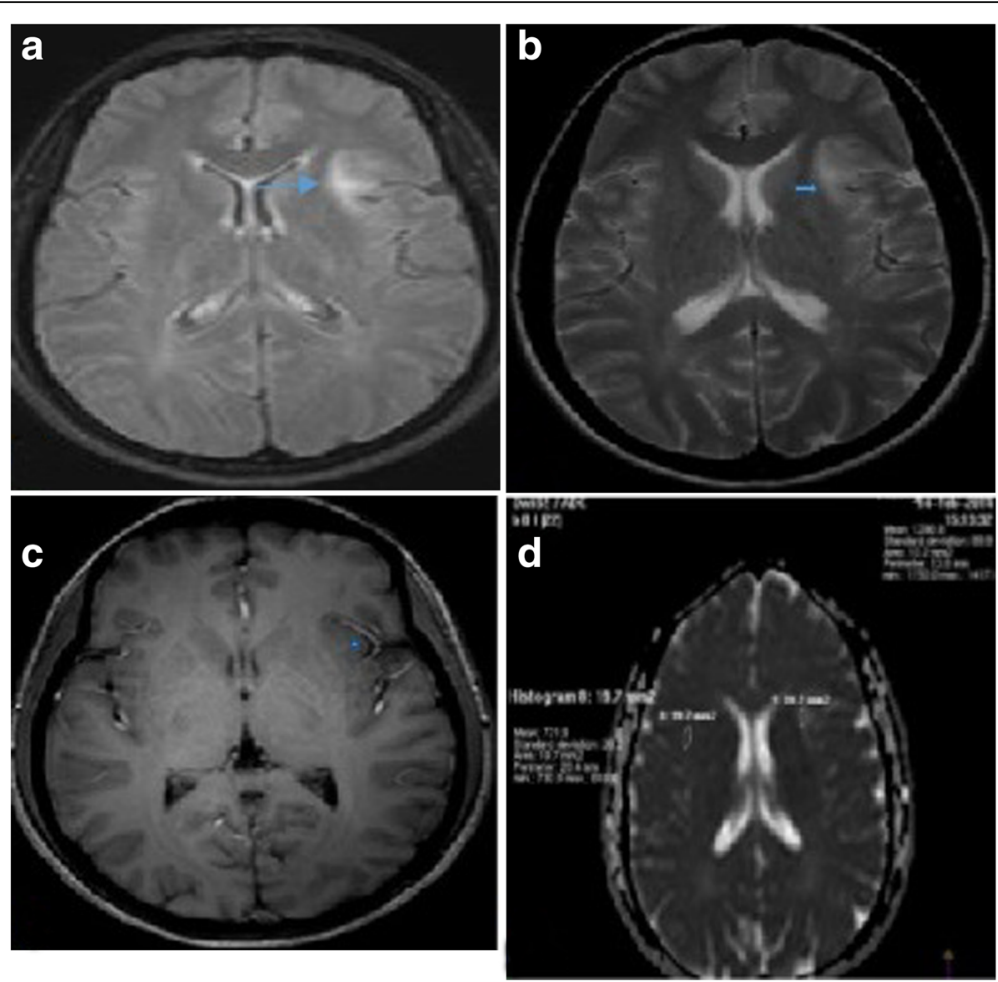

Fig. 5 A 21-year-old female patient with epilepsy. a Subcortical hyperintensity in axial FLAIR sequence. $\mathbf{b}$ Increase in cortical signal intensity in axial T2A sequence and subcortical blurring. $\mathbf{c}$ Cortical thickening in axial T1A sequence. $\mathbf{d}$ Hyperintense area in axial dADC sequence, ADC measurement from this area and from a symmetrical area

could not be assessed due to the lack of a control group. In addition, MR imaging findings relied on conventional visual analysis. For this reason, experienced neuroradiologists were used to diagnose FCD with conventional MRI.

\section{Conclusion}

$\mathrm{SCH}$, blurring, and $\mathrm{CT}$ were the most common findings in our current study. We believe that further investigation of these findings may lead to an easier diagnosis of patients with suspected FCD. All of the lesions in the current study had a diffusion increase in DWI. The quantitative mean ADC values determined via the differential diagnosis of other corticalsubcortical lesions with a diffusion increase can be used as a reference. Detection of conventional MRI findings of FCD, which is resistant to medical treatment, in addition to findings from EEG and dADC values may facilitate the pre-operative diagnosis of FCD.

Table 7 Typical MR imaging findings of FCD type 2 that have been reported in the literature and histologically proven

\begin{tabular}{|c|c|c|c|c|c|c|c|}
\hline & $\begin{array}{l}\text { Urbach et al. } \\
2002[15]\end{array}$ & $\begin{array}{l}\text { Colombo et al. } \\
2003 \text { [18] }\end{array}$ & $\begin{array}{l}\text { Widjaja et al. } \\
2008 \text { [16] }\end{array}$ & $\begin{array}{l}\text { Krsek et al. } \\
2009[14]\end{array}$ & $\begin{array}{l}\text { Lerner et al. } \\
2009 \text { [10] }\end{array}$ & $\begin{array}{l}\text { Mallerio et al. } \\
2012 \text { [22] }\end{array}$ & This study \\
\hline Period of study & $1996-2000$ & $1996-2000$ & $1991-2005$ & $2002-2005$ & $2000-2007$ & 2000-2011 & $2011-2016$ \\
\hline Cortical thickening & $18(81 \%)$ & $10(67 \%)$ & $8(61 \%)$ & $8(50 \%)$ & $43(67 \%)$ & $30(71 \%)$ & $48(\% 76)$ \\
\hline Blurring of GWM junction & $8(36 \%)$ & $13(87 \%)$ & $11(84 \%)$ & $11(69 \%)$ & $47(73 \%)$ & $38(90 \%)$ & $52(\% 82)$ \\
\hline Cortical signal intensity & NA & $6(40 \%)$ & $2(15 \%)$ & $10(62 \%)$ & $22(35 \%)$ & $21(50 \%)$ & $41(\% 65)$ \\
\hline $\begin{array}{l}\text { Subcortical white matter } \\
\text { hyperintensity }\end{array}$ & $22(100 \%)$ & $13(87 \%)$ & $11(84 \%)$ & $14(87 \%)$ & $64(100 \%)$ & $42(100 \%)$ & $52(\% 82)$ \\
\hline Transmantle sign & $18(81 \%)$ & $3(20 \%)$ & $8(72 \%)$ & $6(37 \%)$ & NA & $35(83 \%)$ & $29(\% 46)$ \\
\hline
\end{tabular}


Table 8 The glial malignancy mean ADC values reported in the literature $\times\left(10^{-3} \mathrm{~mm}^{2} / \mathrm{s}\right)$

\begin{tabular}{|c|c|c|c|c|}
\hline & & $\begin{array}{l}\text { Ignjatović J at al. } \\
2015 \text { [31] }\end{array}$ & $\begin{array}{l}\text { Xu Bai et al. } \\
2011 \text { [32] }\end{array}$ & $\begin{array}{l}\text { E.J. Lee et al. } \\
2008 \text { [33] }\end{array}$ \\
\hline \multirow[t]{2}{*}{ Low-grade astrocytoma } & Grade 1 astrocytoma & $0.614 \pm 0.032$ & $0.91 \pm 0.07$ & \\
\hline & Grade 2 astrocytoma & $0.530 \pm 0.114$ & & 1.019 \\
\hline
\end{tabular}

\section{Abbreviations}

ADC: Apparent diffusion coefficient; Blurring: Blurring of the gray-white matter interface; CSF: Cerebrospinal fluid; CSI: Cortical signal increase; $C T$ : Cortical thickening; DWIs: Diffusion-weighted images; EEG: Electroencephalography; FA: Fraction anisotropy; FCD: Focal cortical dysplasia; GWM: Blurring of the gray-white matter; ml: Myoinositol; MRI: Magnetic resonance imaging; MRS: Magnetic resonance spectroscopy; NAA:Cr: N-Acetylaspartate:creatinine ratio; rCBV: Cerebral blood flow; SCH: Subcortical hyperintensity; TMS: Transmantle sign

\section{Acknowledgements}

We confirm that the manuscript has been read and approved by all named authors and that there are no other persons who satisfied the criteria for authorship but are not listed. We further confirm that the order of authors listed in the manuscript has been approved by all of us.

\section{Authors' contributions}

AA designed the article, made the division of labor. MAD checked the suitability of the manuscript for academic language. ZTD participated in the sequence alignment. MT participated in the design of the study and performed the statistical analysis. FE conceived of the study, and participated in its design and coordination and helped to draft the manuscript. All authors read and approved the final manuscript.

\section{Funding}

We wish to confirm that there are no known conflicts of interest associated with this publication and there has been no significant financial support and funding for this work that could have influenced its outcome.

\section{Availability of data and materials}

All data and materials were used in our study and the statistical analysis was done completely. The data and materials mentioned in the study are completely real. No imaginary data and materials were used

\section{Ethics approval and consent to participate}

All procedures performed in the studies involving human participants were in accordance with the ethical standards of the institutional and/or national research committee and with the 1964 Helsinki Declaration and its later amendments or comparable ethical standards.

This study was approved by the ethics committee of our university.

\section{Consent for publication}

We confirm that we have given due consideration to the protection of intellectual property associated with this work and that there are no impediments to publication, including the timing of publication, with respect to intellectual property. In so doing, we confirm that we have followed the regulations of our institutions concerning intellectual property

\section{Competing interests}

The authors declare that they have no competing interests

\section{Author details}

'Department of Radiology, Health Science University Gazi Yaşargil Education Research Hospital, 21090 Diyarbakır, Turkey. ${ }^{2}$ Department of Radiology, Health Science University Van Education Research Hospital, Van, Turkey. ${ }^{3}$ Department of Radiology, Dicle University Medical Faculty, Diyarbakır, Turkey.
Received: 4 July 2019 Accepted: 22 July 2019

Published online: 06 September 2019

\section{References}

1. Taylor DC, Falconar MA, Bruton CJ, Corsellis JA (1971) Focal dysplasia of the cerebral cortex in epilepsy. J Neurol Neurosurg Psychiatry 34:369-387

2. Palmini A, Najm I, Avanzini G et al (2004) Terminology and classification of the cortical dysplasias. Neurology. 62:S2-58

3. Crino PB, Miyata H, Vinters HV (2002) Neurodevelopmental disorders as a cause of seizures: neuropathologic, genetic, and mechanistic considerations. Brain Pathol 12:212-233

4. Cepeda C, Hurst RS, Flores-Hernandez I et al (2003) Morphological and electrophysiological characterization of abnormal cell types in pediatric cortical dysplasia. J Neurosci Res 72(4):472-486

5. Barkovich AJ, Guerrini R, Kuzniecky RI, Jackson GD, Dobyns WB (2012) A developmental and genetic clas-update 2012. Brain 135(Pt 5:1348-1369

6. Mühlebner A, Caros R, Kobow K, Feucht M (2012) Neuropathologic measurements in focal cortical dysplasias:validation of the ILAE 2011 classification system and disgnostic implacations for MRI. Acta Neuropathol 123(2):259-272

7. Fellah S, Callot V, Viout P, Confort G (2012) Epileptogenic brain lesions in children the added of the -value of combined diffusion imaging and proton MR spectroscopy to the presurgical differential diagnosis. Childs Nerv Syst 28(2):273-282

8. Blümcke I, Thom M, Aronica E, Amstrong D (2011) The clinicopathologic spectrum of focal cortical displasias:a concensus classification proposed by an ad hoc task force of the ILAE Diagnostic Methods Commission. Epilepsia 52(1):158-174

9. Chassoux F, Landre E, Mallerio C (2012) Type II focal cortical dysplasia: electroclinical phenotype and surgical outcome related to imaning. Epipesia 52(2):349-358

10. Lerner JT, Salamon N, Hauptman JS et al (2009) Assessment and surgical outcomes for mild type I and severe type II cortical dysplasia: a critical review and the UCLA experience. Epilepsia 50:1310-1335

11. Krsek P, Maton B, Jayakar P et al (2009) Incomplete resection of focal cortical dysplasia is the main predictor of poor postsurgical outcome. Neurology 72:217-212

12. Urbach H, Scheffler B, Heinrichsmeier T et al (2002) Focal cortical dysplasia of Taylor's balloon cell type: a clinicopathological entity with characteristic neuroimaging and histopathological features, and favorable postsurgical outcome. Epilepsia 43:33-40

13. Widjaja E, Nilsson D, Blaser $S$ et al (2008) White matter abnormalities in children with idiopathic developmental delay. Acta Radiol 49:589-595

14. Widdess-Walsh P, Kellinghaus $C$, Jeha $L$ et al (2005) Electro-clinical and imaging characteristics of focal cortical dysplasia: correlation with pathological subtypes. Epilepsy Res 67:25-33

15. Colombo N, Citterio A, Galli C et al (2003) Neuroimaging of focal cortical dysplasia: neuropathological correlations. Epileptic Disord 5(suppl 2):S67-S72

16. Chan S, Chin SS, Nordli DR et al (1998) Prospective magnetic resonance imaging identification of focal cortical dysplasia, including the non-balloon cell subtype. Ann Neurol 44:749-757

17. Kuzniecky R, Morawetz R, Faught E et al (1995) Frontal and central lobe focal dysplasia: clinical, EEG and imaging features. Dev Med Child Neurol 37: 159-166

18. Lee SK, Choe G, Hong KS et al (2001) Neuroimaging findings of cortical dyslamination with cytomegaly. Epilepsia 42:850-856

19. Mackay MT, Becker LE, Chuang SH et al (2003) Malformations of cortical development with balloon cells: clinical and radiologic correlates. Neurology 60:580-587

20. Bernasconi A, Antel SB, Collins DL et al (2001) Texture analysis and morphological processing of magnetic resonance imaging assist detection 
of focal cortical dysplasia in extra-temporal partial epilepsy. Ann Neurol 49: 770-775

21. Colombo N, Tassi L, Galli C et al (2003) Focal cortical dysplasias: MR imaging, histopathologic, and clinical correlations in surgically treated patients with epilepsy. AJNR Am J Neuroradiol 24:724-733

22. Tassi L, Colombo N, Garbelli R et al (2002) Focal cortical dysplasia: neuropathological subtypes, EEG, neuroimaging and surgical outcome. Brain 125 (pt 8:1719-1732

23. Mellerio C, Labeyrie M-A, Chassoux F et al (2012) Optimizing MR imaging detection of type 2 focal cortical dysplasia: best criteria for clinical practice. Am J Neuroradiol 33(10):1932-1938

24. Barkovich AJ, Kuzniecky Rl, Bollen AW et al (1997) Focal transmantle dysplasia: a specific malformation of cortical development. Neurology 49: 1148-1152

25. Lasjaunias P, Manelfe C, Terbrugge K et al (1986) Endovascular treatment of cerebral arteriovenous malformations. Neurosurg Rev 9:265-275

26. Jeha LE, Najm I, Bingaman W et al (2007) Surgical outcome and prognostic factors of frontal lobe epilepsy surgery. Brain 130:574-584

27. McGonigal A, Bartolomei F, Regis J et al (2007) Stereoelectroencephalography in presurgical assessment of MRI-negative epilepsy. Brain 130:3169-3183

28. Chapman K, Wyllie E, Najm I et al (2005) Seizure outcome after epilepsy surgery in patients with normal preoperative MRI. J Neurol Neurosurg Psychiatry 76:710-713

29. Jin G, An N, Jacobs MA, Li K (2010) The role of parallel diffusion-weighted imaging and apparent diffusion coefficient (ADC) map values for evaluating breast lesions: preliminary results. Acad Radiol 17:456-463

30. Englander SA, Ulug AM, Brem R, Glickson JD, van Ziil PC (1997) Diffusion imaging of human breast. NMR Biomed 10:b348-b352

31. Ignjatović J, Stojanov D, Zivković V et al (2015) Apparent diffusion coefficient in the evaluation of cerebral gliomas malignancy. Vojnosanit Pregl 72(10):870-875

32. Bai $X$, Zhang Y, Liu Y (2011) Grading of supratentorial astrocytic tumors by using the difference of ADC value. Neuroradiology. 53(7):533-539

33. Lee EJ, Lee SK, Agid R et al (2008) Preoperative grading of presumptive lowgrade astrocytomas on MR imaging: diagnostic value of minimum apparent diffusion coefficient. AJNR Am J Neuroradiol. 29(10):1872-1877

\section{Publisher's Note}

Springer Nature remains neutral with regard to jurisdictional claims in published maps and institutional affiliations.

\section{Submit your manuscript to a SpringerOpen ${ }^{\circ}$ journal and benefit from:}

- Convenient online submission

- Rigorous peer review

- Open access: articles freely available online

- High visibility within the field

- Retaining the copyright to your article

Submit your next manuscript at $\boldsymbol{\nabla}$ springeropen.com 